PROCEEDINGS OF THE

AMERICAN MATHEMATICAL SOCIETY

Volume 132, Number 12, Pages 3537-3547

S 0002-9939(04)07397-6

Article electronically published on July 14, 2004

\title{
THE DUALITY THEORY OF A FINITE DIMENSIONAL DISCRETE QUANTUM GROUP
}

\author{
LINING JIANG, MAOZHENG GUO, AND MIN QIAN
}

(Communicated by David R. Larson)

\begin{abstract}
Suppose that $\mathcal{H}$ is a finite dimensional discrete quantum group and $K$ is a Hilbert space. This paper shows that if there exists an action $\gamma$ of $\mathcal{H}$ on $L(K)$ so that $L(K)$ is a modular algebra and the inner product on $K$ is $\mathcal{H}$-invariant, then there is a unique $\mathrm{C}^{*}$-representation $\theta$ of $\mathcal{H}$ on $K$ supplemented by the $\gamma$. The commutant of $\theta(\mathcal{H})$ in $L(K)$ is exactly the $\mathcal{H}$ invariant subalgebra of $L(K)$. As an application, a new proof of the classical Schur-Weyl duality theory of type A is given.
\end{abstract}

\section{Preliminaries}

This paper presents a duality theory between a finite dimensional discrete quantum group and its fix-point subalgebra of an operator algebra $L(K)$ where $K$ is a Hilbert space.

As we have known, if $G$ is a finite group with a unit $e$ and $A$ is the algebra of functions from $G$ to $\mathbb{C}$ with pointwise operation, then $A$ can be made into a Hopf algebra if we define comultiplication, counit and antipode by

$$
(\Delta(f))(s, t)=f(s t), \quad \varepsilon(f)=f(e), \quad(S f)(t)=f\left(t^{-1}\right),
$$

where $f \in A$ and $s, t \in G$. We have $\Delta(A) \subseteq A \otimes A$ if we identify $A \otimes A$ with functions on $G \times G$. However this is no longer possible if $G$ is infinite since the range of $\Delta$ is no longer in $A \otimes A$. This leads to the concept of a multiplier Hopf algebra [3], which is the generalized notion of a Hopf algebra such that the above example, with an infinite group, is exactly a multiplier Hopf algebra.

A discrete quantum group [4, which was studied first as duals of compact quantum matrix groups, is a multiplier Hopf *-algebra $(A, \Delta)$ where the algebra $A$ is a direct sum of full matrix algebras over $\mathbb{C}$ with the natural involution. This paper proves that if $\mathcal{H}$ is a finite dimensional discrete quantum group and $L(K)$ is an $\mathcal{H}$ modular algebra where $K$ is a Hilbert space with an $\mathcal{H}$-invariant inner product and $L(K)$ is the algebra of linear bounded operators, then there is a duality between $\mathcal{H}$ and its fix-point subalgebra of $L(K)$.

In fact, this duality property has its inherent physics meaning. Suppose that $G$ is a finite group. The $G$-spin models can provide the simplest examples of lattice field theories exhibiting quantum symmetry. In general, the $G$-spin models with an

Received by the editors November 28, 2001 and, in revised form, December 25, 2002.

2000 Mathematics Subject Classification. Primary 46L05; Secondary 16W30.

Key words and phrases. Discrete quantum group, $\mathrm{C}^{*}$-homomorphism, duality.

This project was supported by the National Natural Science Foundation of China (10301004). 
Abelian group $G$, are known to have a symmetry group $G \times \widetilde{G}$, where $\widetilde{G}$ denotes the Pontryagin dual of $G$ (the group of characters of $G$ ). If $G$ is non-Abelian, the Pontryagin dual $\widetilde{G}$ loses its meaning and the models have a symmetry of a double algebra $D(G)$ [14], which is an algebra defined as the crossed product of $C(G)$ and $\mathbb{C} G$ with the adjoint action of the latter on the former ([2], [5]). In detail, letting $\mathcal{F}$ be the field algebra of a $G$-spin model [14, there is a natural action of $D(G)$ on $\mathcal{F}$ so that $\mathcal{F}$ becomes a $D(G)$-modular algebra. Then the $\mathrm{C}^{*}$-algebra $\mathcal{A}$, which is a $D(G)$-invariant subalgebra of $\mathcal{F}$, is obtained. When an irreducible representation, associated to a $D(G)$-invariant state, $\pi$ of $\mathcal{F}$ is given, there emerges a realization of $D(G)$ so that $D(G)$ and $\pi(\mathcal{A})$ are exactly the commutants of each other. It is easy to see that the double algebra $D(G)$ of $G$ is a discrete quantum group, and this example fits into the scheme.

Besides the duality theory in $G$-spin models, there are many duality results; for example, the Schur-Weyl duality between the symmetric group and the general linear group ([6], [15]), the Jimbo-Schur-Weyl duality between the quantum group of type A and Hecke ([7], [8]), and so on, fit into this scheme. As an application of the duality theorem, not using the highest weight representation of the general linear group anymore, we give a new proof of the classical Schur-Weyl duality of type A. Notice that the discrete quantum group in this paper is finite dimensional. As to the infinite dimensional case, it is under consideration now.

All algebras in this paper will be *-algebras over the complex field $\mathbb{C}$. For general results on Hopf algebras one can refer to the books of Abe [1] and Sweedler [13. We shall use their notation; so we shall use $m, \Delta, \varepsilon$ and $S$ for the multiplication, the comultiplication, the counit and the antipode respectively. Also we shall adopt the summation convention, which is standard in Hopf algebra theory:

$$
\triangle(a)=\sum_{(a)} a_{(1)} \otimes a_{(2)}
$$

and so on. The formula $m \circ(i d \otimes S) \circ \triangle(a)=\varepsilon(a) 1$ can be written, for example, as

$$
\sum_{(a)} a_{(1)} S\left(a_{(2)}\right)=\varepsilon(a) 1
$$

\section{Construction of a $\mathrm{C}^{*}$-Algebra Related to a finite dimensional DISCRETE QUANTUM GROUP}

Let us recall the definition of a discrete quantum group.

Definition 2.1 ([3, 4]). A discrete quantum group is a pair $(A, \Delta)$ where $A$ is a direct sum of full matrix algebras with the natural ${ }^{*}$-structure and $\Delta$ is a comultiplication on $A$ making $A$ into a multiplier Hopf *-algebra.

The discrete quantum group was studied first as a dual of a compact quantum matrix group [12]. It is clear that if $G$ is a finite group, the group algebra $\mathbb{C} G$ and the double algebra $D(G)$ of $G$ are both discrete quantum groups.

In this paper, let $\mathcal{H}$ be a finite dimensional discrete quantum group. One can suppose that $\mathcal{H}=\sum_{\alpha \in I} \oplus A_{\alpha}$ where $I$ is a finite index set and $A_{\alpha}$ are full matrix algebras. There is a unique $C^{*}$-norm in $\mathcal{H}$ so that it becomes a $C^{*}$-algebra. Also [4] is a general involutive Hopf algebra (i.e. $\mathcal{H}$ is a Hopf algebra and $S^{2}=i d$ ) since 
it has a unit 1 and a unique element $z$ (call it an integral) satisfying $z=z^{*}=z^{2}$, $\varepsilon(z)=1$ and $\forall h \in \mathcal{H}$,

$$
h z=z h=\varepsilon(h) z .
$$

Without loss of generality, one can suppose that

$$
\Delta(z)=\sum_{\alpha \in I} u_{\alpha} \otimes v_{\alpha}
$$

where $u_{\alpha} \in A_{\alpha}$. Then

Lemma 2.1. $v_{\alpha}=S\left(u_{\alpha}^{*}\right)$.

Proof. Using Proposition 4.3 of [4], the lemma is clear.

Definition 2.2. Suppose that $A$ is an algebra. We call $A$ an $\mathcal{H}$-modular algebra if there is a linear action $\gamma$ of $\mathcal{H}$ on $A$ so that

$$
\begin{aligned}
\gamma(a)(F G) & =\sum_{(a)} \gamma\left(a_{(1)}\right)(F) \gamma\left(a_{(2)}\right)(G), \\
\gamma(a b)(G) & =\gamma(a)(\gamma(b)(G)),
\end{aligned}
$$

and that

$$
\gamma(a)\left(G^{*}\right)=\gamma\left(S\left(a^{*}\right)\right)(G)^{*} \quad \text { (conjugate property) }
$$

where $a, b \in \mathcal{H}, \Delta(a)=\sum_{(a)} a_{(1)} \otimes a_{(2)}$ and $F, G \in A$.

In the following, suppose that $K$ is a Hilbert space and $L(K)$, the algebra of all linear bounded operators, is an $\mathcal{H}$-modular algebra. Also, by $\gamma_{a}(a \in A)$ we denote the mapping $\gamma(a)$.

Proposition 2.2. Set

$$
\mathcal{A}=\left\{T \in L(K): \gamma_{z}(T)=T\right\} .
$$

Then $\mathcal{A}$ is a nonzero *-algebra.

Proof. Since $S\left(z^{*}\right)=z$, and $\forall T \in L(K)$,

$$
\gamma_{z}\left(T^{*}\right)=\gamma_{z}(T)^{*}
$$

then $\mathcal{A}$ is closed under the ${ }^{*}$-operation. Now it suffices to prove that $\mathcal{A}$ is an algebra. Indeed, $\forall F, G \in L(K)$,

$$
\begin{aligned}
\gamma_{z}\left(\gamma_{z}(F) \gamma_{z}(G)\right) & =\sum_{(z)} \gamma_{z_{(1)}}(F) \gamma_{z_{(2)}}(G) \\
& =\sum_{(z)} \varepsilon\left(z_{(1)} \varepsilon\left(z_{(2)}\right)\right) \gamma_{z}(F) \gamma_{z}(G) \\
& =\gamma_{z}(F) \gamma_{z}(G)
\end{aligned}
$$

This implies that $\forall \gamma_{z}(F), \gamma_{z}(G) \in \mathcal{A}, \gamma_{z}(F) \gamma_{z}(G) \in \mathcal{A}$. Therefore $\mathcal{A}$ is an algebra.

Remark 2.1. It is easy to see that

$$
\mathcal{A}=\left\{T \in L(K): \gamma_{a}(T)=\varepsilon(a) T \quad \forall a \in \mathcal{H}\right\} .
$$

In other words, $\mathcal{A}$ is an $\mathcal{H}$-invariant subalgebra of $L(K)$.

Theorem 2.3. $\mathcal{A}$ is a $C^{*}$-algebra. 
The proof involves several steps, which are of interest in their own right.

Lemma 2.4 ([10]). Suppose that $A$ is a $C^{*}$-algebra with a unit 1 . If there exists a positive linear map $P: A \rightarrow A$ satisfying $P(1)=1$ and for $\forall a, b_{1}, b_{2} \in A$,

$$
P\left(P\left(b_{1}\right) a P\left(b_{2}\right)\right)=P\left(b_{1}\right) P(a) P\left(b_{1}\right) \quad \text { (bi-modular property), }
$$

then $P$ is a projection of norm 1 from $A$ onto $P(A):=B$. (Such a projection is often called a conditional expectation from $A$ onto $B$.)

Lemma 2.5. $\gamma_{z}$ is a conditional expectation.

Proof. 1) The map $\gamma_{z}: L(K) \rightarrow \mathcal{A}$ is positive. Indeed $\forall G \in L(K)$,

$$
\begin{aligned}
\gamma_{z}\left(G G^{*}\right) & =\sum_{(z)} \gamma_{z_{(1)}}(G) \gamma_{z_{(2)}}\left(G^{*}\right) \quad(\text { by }(2.3)) \\
& =\sum_{\alpha \in I} \gamma_{u_{\alpha}}(G) \gamma_{v_{\alpha}}\left(G^{*}\right) \quad(\text { by }(2.2)) \\
& =\sum_{\alpha \in I} \gamma_{u_{\alpha}}(G)\left(\gamma_{S\left(v_{\alpha}^{*}\right)}(G)\right)^{*}(\text { by }(2.5)) \\
& =\sum_{\alpha \in I} \gamma_{u_{\alpha}}(G)\left(\gamma_{u_{\alpha}}(G)\right)^{*} \text { by (Lemma 2.1). }
\end{aligned}
$$

Since the sum of positive elements is also a positive element, $\gamma_{z}\left(G G^{*}\right)$ is a positive operator and thus $\gamma_{z}$ is a positive linear map.

2) (bi-modular property) $\forall G_{1}, G_{2}, G_{3} \in L(K)$,

$$
\begin{aligned}
\gamma_{z}\left(\gamma_{z}\left(G_{1}\right) G_{2} \gamma_{z}\left(G_{3}\right)\right) & =\sum_{(z)} \gamma_{z_{(1)}}\left(\gamma_{z}\left(G_{1}\right)\right) \gamma_{z_{(2)}}\left(G_{2}\right) \gamma_{z_{(3)}}\left(\gamma_{z}\left(G_{3}\right)\right) \\
& =\sum_{(z)} \gamma_{z_{(1)} z}\left(G_{1}\right) \gamma_{z_{(2)}}\left(G_{2}\right) \gamma_{z_{(3)} z}\left(G_{3}\right) \\
& =\sum_{(z)} \varepsilon\left(z_{(1)}\right) \gamma_{z}\left(G_{1}\right) \gamma_{z_{(2)}}\left(G_{2}\right) \gamma_{z_{(3)} z}\left(G_{3}\right) \\
& =\sum_{(z)} \gamma_{z}\left(G_{1}\right) \gamma_{\varepsilon\left(z_{(1)}\right) z_{(2)}}\left(G_{2}\right) \gamma_{z_{(3)}}\left(G_{3}\right) \\
& =\sum_{(z)} \gamma_{z}\left(G_{1}\right) \gamma_{z_{(1)}}\left(G_{2}\right) \gamma_{z_{(2)}}\left(G_{3}\right) \\
& =\sum_{(z)} \gamma_{z}\left(G_{1}\right) \gamma_{z_{(1)} \varepsilon\left(z_{(2)}\right)}\left(G_{2}\right) \gamma_{z}\left(G_{3}\right) \\
& =\gamma_{z}\left(G_{1}\right) \gamma_{z}\left(G_{2}\right) \gamma_{z}\left(G_{3}\right) .
\end{aligned}
$$

Thus $\gamma_{z}$ is a conditional expectation since $\gamma_{z}(1)=1$ is clear. By Lemma 2.4, $\gamma_{z}$ is a projection of norm one from $L(K)$ onto $\mathcal{A}$.

Now we give the proof of Theorem 2.3.

Proof. In order to prove that $\mathcal{A}$ is a $C^{*}$-algebra, it suffices to prove $\mathcal{A}$ is closed under the operator norm topology. Indeed, suppose that $\exists x_{n} \in \mathcal{A}: \lim _{n \rightarrow \infty} x_{n}=x$, i.e. 
$x \in \overline{\mathcal{A}}$, the norm closure of $\mathcal{A}$ in $L(K)$. Since $\gamma_{z}$ is a bounded projection,

$$
\begin{aligned}
x & =\lim _{n \rightarrow \infty} x_{n} \\
& =\lim _{n \rightarrow \infty} \gamma_{z}\left(x_{n}\right) \\
& =\gamma_{z}\left(\lim _{n \rightarrow \infty} x_{n}\right) \\
& =\gamma_{z}(x) .
\end{aligned}
$$

That is to say, $x=\gamma_{z}(x) \in \mathcal{A}$, and $\mathcal{A}$ is completed under the $C^{*}$-norm. Therefore $\mathcal{A}$ is a $C^{*}$-algebra.

\section{The duality between $\mathcal{A}$ and $\mathcal{H}$}

This section will build a duality between $\mathcal{A}$ and $\mathcal{H}$ on the Hilbert space $K$.

Proposition 3.1. Suppose that the inner-product (, ) on $K$ is $\mathcal{H}$-invariant, i.e., there is a vector $\Omega \in K$ of norm one so that $\forall a \in \mathcal{H}, T \in L(K)$,

$$
\left(\Omega, \gamma_{a}(T) \Omega\right)=\varepsilon(a)(\Omega, T \Omega) .
$$

Then there exists a unique $C^{*}$-homomorphism $\theta: \mathcal{H} \rightarrow L(K)$ with the following two properties: $\forall a \in \mathcal{H}, T \in L(K)$,

$$
\begin{aligned}
\gamma_{a}(T)=\sum_{(a)} \theta\left(a_{(1)}\right) T \theta\left(S a_{(2)}\right), \\
\theta(a) \Omega=\varepsilon(a) \Omega .
\end{aligned}
$$

Proof. 1) We first construct a $C^{*}$-homomorphism $\theta: \mathcal{H} \rightarrow L(K)$. Since $\mathcal{H}$ is involutive, i.e. $S^{2}=i d$, by [9, for all $h \in \mathcal{H}$,

$$
\sum_{(h)} S\left(h_{(2)}\right) h_{(1)}=\sum_{(h)} h_{(2)} S\left(h_{(1)}\right)=\varepsilon(h) 1,
$$

where 1 denotes the unit of $\mathcal{H}$. Then $\forall P, T \in L(K)$ and $a \in \mathcal{H}$,

$$
\begin{aligned}
& \left(P \Omega, \gamma_{a}(T) \Omega\right)=\left(\Omega, P^{*}\left(\gamma_{a}(T) \Omega\right)\right) \\
& =\sum_{(a)}\left(\Omega, \overline{\varepsilon\left(a_{(2)}\right)} P^{*}\left(\gamma_{a_{(1)}}(T) \Omega\right)\right) \\
& =\sum_{(a)}\left(\Omega, P^{*}\left(\gamma_{a_{(1)} \varepsilon\left(a_{(2)}\right)}(T) \Omega\right)\right) \\
& =\sum_{(a)} \varepsilon\left(S\left(a_{(2)}\right)\right)\left(\Omega, P^{*}\left(\gamma_{a_{(1)}}(T) \Omega\right)\right) \quad(\varepsilon=\varepsilon \circ S) \\
& =\sum_{(a)}\left(\Omega, \gamma_{S\left(a_{(2)}\right)}\left(P^{*} \gamma_{a_{(1)}}(T)\right) \Omega\right) \quad \text { (by (3.1)) } \\
& =\sum_{(a)}\left(\Omega, \gamma_{S\left(a_{(3)}\right)}\left(P^{*}\right) \gamma_{S\left(a_{(2)}\right) a_{(1)}}(T) \Omega\right) \quad(\text { by }(2.3)) \\
& =\sum_{(a)}\left(\Omega,\left(\gamma_{a_{(3)}^{*}}(P)\right)^{*} \gamma_{S\left(a_{(2)}\right) a_{(1)}}(T) \Omega\right) \quad(\text { by }(2.5)) \\
& =\sum_{(a)} \overline{\varepsilon\left(a_{(1)}\right)}\left(\Omega,\left(\gamma_{a_{(2)}^{*}}(P)\right)^{*} \gamma_{1}(T) \Omega\right) \quad(\text { by }(3.4))
\end{aligned}
$$




$$
\begin{aligned}
& =\sum_{(a)}\left(\Omega,\left(\gamma \gamma_{\varepsilon\left(a_{(1)}\right)} a_{(2)}^{*}(P)\right)^{*} T \Omega\right) \\
& =\left(\Omega,\left(\gamma_{a^{*}}(P)\right)^{*} T \Omega\right) \\
& =\left(\gamma_{a^{*}}(P) \Omega, T \Omega\right),
\end{aligned}
$$

where for $x \in \mathbb{C}, \bar{x}$ means the complex conjugate of $x$. We therefore have

$$
\left(P \Omega, \gamma_{a}(T) \Omega\right)=\left(\gamma_{a^{*}}(P) \Omega, T \Omega\right)
$$

and have $\gamma_{a}(T) \Omega=0$ if $T \Omega=0$. Thus $\forall a \in \mathcal{H}$ the linear map

$$
\theta(a): T \Omega \rightarrow \gamma_{a}(T) \Omega
$$

is well defined since $L(K) \Omega=K$. By (3.6), it is easy to see that $\theta\left(a^{*}\right)=\theta(a)^{*}$. Now to see that $\theta: \mathcal{H} \rightarrow L(K)$ is a $C^{*}$-homomorphism, it suffices to see that $\theta(a)$ is continuous. Indeed, suppose that $T_{n}, T \in L(K)$ with $T_{n} \Omega \rightarrow T \Omega . \forall P \in L(K)$,

$$
\begin{aligned}
\lim _{n \rightarrow \infty}\left(P \Omega, \gamma_{a}\left(T_{n}\right) \Omega\right) & =\lim _{n \rightarrow \infty}\left(\gamma_{a^{*}}(P) \Omega, T_{n} \Omega\right) \\
& =\left(\gamma_{a^{*}}(P) \Omega, T \Omega\right) \\
& =\left(P \Omega, \gamma_{a}(T) \Omega\right)
\end{aligned}
$$

By the principle of uniform boundedness, $\lim _{n \rightarrow \infty} \gamma_{a}\left(T_{n}\right) \Omega=\gamma_{a}(T) \Omega$. Thus $\theta(a)$ is continuous.

2) The representation $\theta: \mathcal{H} \rightarrow L(K)$ has properties (3.2) and (3.3). Indeed, (3.3) is clear. As to (3.2), $\forall T, G \in L(K)$,

$$
\begin{aligned}
\sum_{(a)} \theta\left(a_{(1)}\right) T \theta\left(S\left(a_{(2)}\right)\right)(G \Omega) & =\sum_{(a)} \theta\left(a_{(1)}\right) T \gamma_{S\left(a_{(2)}\right)}(G) \Omega \quad(\text { by }(3.8)) \\
& =\sum_{(a)} \gamma_{a_{(1)}}\left(T \gamma_{S\left(a_{(2)}\right)}(G)\right) \Omega \quad(\text { by }(3.8)) \\
& =\sum_{(a)} \gamma_{a_{(1)}}(T) \gamma_{a_{(2)} S\left(a_{(3)}\right)}(G) \Omega \quad(\text { by }(2.3)) \\
& =\sum_{(a)} \gamma_{a_{(1)}}(T) \gamma_{\varepsilon\left(a_{(2)}\right)}(G) \Omega \\
& =\sum_{(a)} \gamma_{a_{(1)} \varepsilon\left(a_{(2)}\right)}(T)(G \Omega) \\
& =\gamma_{a}(T)(G \Omega) .
\end{aligned}
$$

Thus $\forall a \in \mathcal{H}, T \in L(K), \sum_{(a)} \theta\left(a_{(1)}\right) T \theta\left(S a_{(2)}\right)=\gamma_{a}(T)$. 
3) (Uniqueness) If there exists another $C^{*}$-homomorphism $\theta^{\prime}: \mathcal{H} \rightarrow L(K)$ with properties (3.2) and (3.3), then $\forall T \in L(K)$,

$$
\begin{aligned}
\theta(a)(T \Omega) & =\gamma_{a}(T) \Omega \\
& =\sum_{(a)} \theta^{\prime}\left(a_{(1)}\right) T \theta^{\prime}\left(S a_{(2)}\right) \Omega \\
& =\sum_{(a)} \theta^{\prime}\left(a_{(1)}\right) T \varepsilon\left(S\left(a_{(2)}\right)\right) \Omega \\
& =\sum_{(a)} \theta^{\prime}\left(a_{(1)}\right) T \varepsilon\left(a_{(2)}\right) \Omega \\
& =\sum_{(a)} \theta^{\prime}\left(\varepsilon\left(a_{(2)}\right) a_{(1)}\right)(T \Omega) \\
& =\theta^{\prime}(a)(T \Omega) .
\end{aligned}
$$

This means $\forall a \in \mathcal{H}, \theta^{\prime}(a)=\theta(a)$. Thus $\theta^{\prime}=\theta$ and we complete the proof.

Using the mapping $\theta$, we can build our main result, which states that there is a duality between $\mathcal{H}$ and $\mathcal{A}$.

Theorem 3.2. Assumptions and notation as in Proposition 3.1. Then,

$$
\theta(\mathcal{H})^{\prime}=\mathcal{A} ; \quad \theta(\mathcal{H})=\mathcal{A}^{\prime},
$$

where the prime denotes the commutant in $L(K)$.

Proof. First, $\forall a \in \mathcal{H}, P=\gamma_{z}(P) \in \mathcal{A}$ and $G \in L(K)$, since

$$
\begin{array}{rlr}
\theta(a) P(G \Omega) & =\gamma_{a}(P G) \Omega & (\text { by }(3.3)) \\
& =\sum_{(a)} \gamma_{a_{(1)}}(P) \gamma_{a_{(2)}}(G) \Omega & \quad \text { by }(2.3)) \\
& =\sum_{(a)} \varepsilon\left(a_{(1)}\right) P \gamma_{a_{(2)}}(G) \Omega & \\
& =\sum_{(a)} P \gamma_{\varepsilon\left(a_{(1)}\right) a_{(2)}}(G) \Omega & \\
& =P \gamma_{a}(G) \Omega \\
& =P \theta(a)(G \Omega),
\end{array}
$$

we have $\theta(a) P=P \theta(a)$. This implies that $\theta(\mathcal{H}) \mathcal{A}=\mathcal{A} \theta(\mathcal{H})$ and that

$$
\theta(\mathcal{H})^{\prime} \supseteq \mathcal{A}^{-} ; \quad \theta(\mathcal{H})^{-} \subseteq \mathcal{A}^{\prime}
$$

where the bar means the weak closure. On the other hand, supposing that $P \in$ $\theta(\mathcal{H})^{\prime}$, i.e. $\theta(a) P=P \theta(a)(\forall a \in \mathcal{H})$, we have

$$
\begin{aligned}
\gamma_{a}(P) & =\sum_{(a)} \theta\left(a_{(1)}\right) P \theta\left(S\left(a_{(2)}\right)\right) \quad(\text { by }(3.2)) \\
& =\sum_{(a)} P \theta\left(a_{(1)} S\left(a_{(2)}\right)\right) \\
& =\varepsilon(a) P .
\end{aligned}
$$

Thus by (2.7), $P \in \mathcal{A}$ and $\theta(\mathcal{H})^{\prime} \subseteq \mathcal{A}$. Therefore $\theta(\mathcal{H})^{\prime}=\mathcal{A}$ and $\theta(\mathcal{H})^{-}=\mathcal{A}^{\prime}$. Since $\operatorname{dim} \mathcal{H}<\infty, \theta(\mathcal{H})^{-}=\theta(\mathcal{H})$, the second equality of Theorem 3.2 is clear. 
Remark 3.1. Under the conditions in Proposition 3.1, $\mathcal{A}$ is a Von Neumann algebra since $\mathcal{A}=\theta(\mathcal{H})^{\prime}$. Also, from the above duality theorem, one can see that the irreducible representations of $\mathcal{A}$ are in one-to-one correspondence with the irreducible representations of $\theta(\mathcal{H})$. However, for a given $\mathcal{H}$-modular algebra $L(K)$, one in general could not get all irreducible representations of $\mathcal{H}$ in $L(K)$. The next section, which will get the classical Schur-Weyl duality of type A, can illustrate this point as well.

Furthermore, assume that $\mathcal{F}$ is a $C^{*}$-algebra and that there is a continuous action $\gamma$ of $\mathcal{H}$ on $\mathcal{F}$ so that $\mathcal{F}$ becomes an $\mathcal{H}$-module algebra.

Corollary 3.3. Suppose that $\pi$ is an irreducible representation of $\mathcal{F}$ on a Hilbert space $K=(\pi(\mathcal{F}) \Omega)^{-}$with a vacuum vector $\Omega$ giving rise to an $\mathcal{H}$-invariant state on $K: \forall a \in \mathcal{H}, G \in \mathcal{F}$,

$$
\left(\Omega, \pi\left(\gamma_{a}(G)\right) \Omega\right)=\varepsilon(a)(\Omega, \pi(G) \Omega) .
$$

If $\forall a \in \mathcal{H}, \exists \lambda>0$ so that $\left\|\gamma_{a}(T) \Omega\right\| \leq \lambda\|T \Omega\|(T \in L(K))$, then there exists a unique $C^{*}$-homomorphism $\theta: \mathcal{H} \rightarrow L(K)$ with the following properties: $\forall a \in \mathcal{H}$, $F \in \mathcal{F}$,

$$
\begin{aligned}
\pi\left(\gamma_{a}(T)\right) & =\sum_{(a)} \theta\left(a_{(1)}\right) \pi(T) \theta\left(S a_{(2)}\right), \\
\theta(a) \Omega & =\varepsilon(a) \Omega,
\end{aligned}
$$

and satisfies the equalities

$$
\theta(\mathcal{H})^{\prime}=\pi(\mathcal{A})^{-} ; \quad \theta(\mathcal{H})=\pi(\mathcal{A})^{\prime} .
$$

Proof. Since the representation $\pi$ of $\mathcal{F}$ is irreducible, $\pi(\mathcal{F})^{-}=L(K)$. The proof is similar to that of Theorem 3.2 and we omit it here.

\section{EXAmples RELATED TO THE SYMMETRIC GROUP}

This section gives an application of Theorem 3.2. Let $S_{m}$ be the symmetric group on the set $\{1,2, \cdots, m\}$ and $\mathbb{C} S_{m}$ the group algebra of $S_{m}$. Then $\mathbb{C} S_{m}$ is a finite discrete quantum group with $g^{*}=g^{-1}$ and a unique integral $z=\frac{1}{m !} \sum_{g \in S_{m}} g$. There is a natural representation $\rho$ of $S_{m}$ on the linear space $\left(\mathbb{C}^{n}\right)^{\otimes m}$ : for $\sigma \in S_{m}$, $v_{i_{1}} \otimes v_{i_{2}} \otimes \cdots \otimes v_{i_{m}} \in\left(\mathbb{C}^{n}\right)^{\otimes m}$,

$$
\rho(\sigma)\left(v_{i_{1}} \otimes v_{i_{2}} \otimes \cdots \otimes v_{i_{m}}\right)=v_{i_{\sigma(1)}} \otimes v_{i_{\sigma(2)}} \otimes \cdots \otimes v_{i_{\sigma(m)}}
$$

where $1 \leq i_{j} \leq n$ and $\left\{v_{1}, v_{2}, \cdots, v_{n},\right\}$ is a linear basis of $\mathbb{C}^{n}$. One can induce an action $\gamma$ of $S_{m}$ on $\operatorname{End}\left(\left(\mathbb{C}^{n}\right)^{\otimes m}\right)$ implemented by $\rho$ : for $\sigma \in S_{m}, T \in \operatorname{End}\left(\left(\mathbb{C}^{n}\right)^{\otimes m}\right)$ $\cong\left(\operatorname{End} \mathbb{C}^{n}\right)^{\otimes m}$,

$$
\gamma_{\sigma}: T \rightarrow \rho(\sigma) \circ T \circ \rho\left(\sigma^{-1}\right) .
$$

Such an action can be extended linearly to $\mathbb{C} S_{m}$ and denoted by $\gamma$ too. Clearly under the action $\gamma, \operatorname{End}\left(\left(\mathbb{C}^{n}\right)^{\otimes m}\right)$ is a $\mathbb{C} S_{m}$-modular algebra. Denote $\gamma_{z}\left(\operatorname{End}\left(\mathbb{C}^{n}\right)^{\otimes m}\right)$ by $\mathcal{A}$. By Theorem $3.2, \mathcal{A}=\left(\rho\left(S_{m}\right)\right)^{\prime}$. On the other hand, let $G L(n)$ be the general linear group of order $n$, and let $\pi^{\prime}$ be the natural representation of the group $G L(n)$ on the space $\mathbb{C}^{n}$. Clearly $\pi^{\prime}(G L(n))$ is the set of all invertible operators in 
$\operatorname{End}\left(\mathbb{C}^{n}\right)$ and $\pi^{\prime}$ can be extended to a representation $\pi$ of the group $G L(n)$ on the space $\left(\mathbb{C}^{n}\right)^{\otimes m}: \forall g \in G L(n)$,

$$
\pi(g)=\pi^{\prime}(g) \otimes \pi^{\prime}(g) \otimes \cdots \otimes \pi^{\prime}(g) .
$$

In this section we will prove $\mathcal{A}=\pi(G L n))^{\prime \prime}$, the algebra generated by the set $\{\pi(g): g \in G L(n)\}$. Namely, we will give a new proof of the well-known classical Schur-Weyl duality of type A.

In the following, by $\sigma_{i}(1 \leq i \leq m-1)$ we mean the generator $(i, i+1)$ of $S_{m}$. $\forall \sigma \in S_{m}, \sigma$ can be decomposed as $\sigma_{i_{1}} \sigma_{i_{2}} \cdots \sigma_{i_{s}}$.

Lemma 4.1. $\pi(G L n))^{\prime \prime} \subseteq \mathcal{A}$.

Proof. It suffices to prove that $\forall T \in \operatorname{End}(\mathbb{C})^{n}, \gamma_{z}\left(T^{\otimes m}\right)=T^{\otimes m}$. First, since $\sigma_{i}^{-1}=\sigma_{i}, \forall v_{k_{1}} \otimes v_{k_{2}} \otimes \cdots \otimes v_{k_{m}} \in\left(\mathbb{C}^{n}\right)^{\otimes m}$,

$$
\begin{aligned}
\gamma_{\sigma_{i}} & \left(T^{\otimes m}\right)\left(v_{k_{1}} \otimes \cdots \otimes v_{k_{m}}\right)=\rho\left(\sigma_{i}\right) \circ T^{\otimes m} \circ \rho\left(\sigma_{i}\right)\left(v_{k_{1}} \otimes \cdots \otimes v_{k_{m}}\right) \\
& =\rho\left(\sigma_{i}\right) \circ T^{\otimes m}\left(v_{k_{1}} \otimes \cdots \otimes v_{k_{i+1}} \otimes v_{k_{i}} \otimes \cdots \otimes v_{k_{m}}\right) \\
& =\rho\left(\sigma_{i}\right)\left(T\left(v_{k_{1}}\right) \otimes \cdots \otimes T\left(v_{k_{i+1}}\right) \otimes T\left(v_{k_{i}}\right) \otimes \cdots \otimes T\left(v_{k_{m}}\right)\right) \\
& =T\left(v_{k_{1}}\right) \otimes \cdots \otimes T\left(v_{k_{i}}\right) \otimes T\left(v_{k_{i+1}}\right) \otimes \cdots \otimes T\left(v_{k_{m}}\right) \\
& =T^{\otimes m}\left(v_{k_{1}} \otimes \cdots \otimes v_{k_{m}}\right) .
\end{aligned}
$$

Thus $\forall 1 \leq i \leq m-1, \gamma_{\sigma_{i}}\left(T^{\otimes m}\right)=T^{\otimes m}$. So, for $\sigma=\sigma_{i_{1}} \sigma_{i_{2}} \cdots \sigma_{i_{s}} \in S_{m}$,

$$
\begin{aligned}
\gamma_{\sigma}\left(T^{\otimes m}\right) & =\sigma \circ T^{\otimes m} \circ \sigma^{-1} \\
& =\left(\sigma_{i_{1}} \sigma_{i_{2}} \cdots \sigma_{i_{s}}\right) \circ T^{\otimes m} \circ\left(\sigma_{i_{1}} \sigma_{i_{2}} \cdots \sigma_{i_{s}}\right)^{-1} \\
& =\left(\sigma_{i_{1}} \sigma_{i_{2}} \cdots \sigma_{i_{s}}\right) \circ T^{\otimes m} \circ\left(\sigma_{i_{s}} \cdots \sigma_{i_{2}} \sigma_{i_{1}}\right) \\
& =\left(\sigma_{i_{1}} \sigma_{i_{2}} \cdots \sigma_{i_{s-1}}\right)\left(\sigma_{i_{s}} \circ T^{\otimes m} \circ \sigma_{i_{s}}\right)\left(\sigma_{i_{s-1}} \cdots \sigma_{i_{2}} \sigma_{i_{1}}\right) \\
& =\cdots \\
& =T^{\otimes m} .
\end{aligned}
$$

Therefore,

$$
\begin{aligned}
\gamma_{z}\left(T^{\otimes m}\right) & =\frac{1}{m !} \sum_{\sigma \in S_{m}} \gamma_{\sigma}\left(T^{\otimes m}\right) \\
& =T^{\otimes m}
\end{aligned}
$$

and $\pi(G L n))^{\prime \prime} \subseteq \mathcal{A}$.

Lemma 4.2. Suppose that $T_{i} \in \operatorname{End}\left(\mathbb{C}^{n}\right)$. Then

$$
\gamma_{z}\left(T_{1} \otimes T_{2} \otimes \cdots \otimes T_{m}\right)=\frac{1}{m !} \sum_{i=1}^{m}\left(\sum_{I \subset\{1,2, \cdots, m\},|I|=i}(-1)^{m-i}\left(\sum_{r \in I} T_{r}\right)^{\otimes m}\right) .
$$

Proof. Similar to the proof of Lemma 4.1, one can see $\forall \sigma \in S_{m}$,

$$
\gamma_{\sigma}\left(T_{1} \otimes T_{2} \otimes \cdots \otimes T_{m}\right)=T_{\sigma(1)} \otimes T_{\sigma(2)} \otimes \cdots \otimes T_{\sigma(m)}
$$


and

$$
\begin{aligned}
\gamma_{z}\left(T_{1} \otimes T_{2} \otimes \cdots \otimes T_{m}\right) & =\frac{1}{m !} \sum_{\sigma \in S_{m}} \gamma_{\sigma}\left(T_{1} \otimes T_{2} \otimes \cdots \otimes T_{m}\right) \\
& =\frac{1}{m !} \sum_{\sigma \in S_{m}} T_{\sigma(1)} \otimes T_{\sigma(2)} \otimes \cdots \otimes T_{\sigma(m)} .
\end{aligned}
$$

Now by a tedious but very straightforward calculation, the result is clear.

Theorem 4.3. $\pi(G L n))^{\prime \prime}=\mathcal{A}$.

Proof. We need only to prove $\mathcal{A} \subseteq \pi(G L n))^{\prime \prime}$. To show this, it suffices to show that $\left.\forall T_{i} \in \operatorname{End}\left(\mathbb{C}^{n}\right)(1 \leq i \leq n), \gamma_{z}\left(T_{1} \otimes T_{2} \otimes \cdots \otimes T_{m}\right) \in \pi(G L n)\right)^{\prime \prime}$.

By [1], $\forall T \in \operatorname{End}\left(\mathbb{C}^{n}\right), T$ can be represented as

$$
T=\sum_{i=1}^{4} t_{i} Q_{i}
$$

where $t_{i} \in \mathbb{C}$ and $Q_{i}$ are positive operators. Namely, $Q_{i}$ are Hermitian elements and $\sigma\left(Q_{i}\right)$, the spectral sets of $Q_{i}$, are contained in $\mathbb{R}^{+} \cup\{0\}$. Set $S_{i}=Q_{i}+I$ $(1 \leq i \leq 4)$ and $S_{5}=\left(-\sum_{i=1}^{4} t_{i}\right) I$. Then by the spectral mapping theorem [11], $S_{i}, S_{i}+S_{j}$ are all invertible positive elements since the sum of any two positive operators is also a positive operators. Thus $T$ can be decomposed as

$$
T=\sum_{i=1}^{5} m_{i} S_{i}
$$

where $m_{i} \in \mathbb{C}$ and $S_{i}$ are all invertible positive elements (and necessary in $G L(n)$ ). Based on this, set

$$
T_{i}=\sum_{k=1}^{5} m_{i_{k}} S_{i_{k}}
$$

where $m_{i_{k}} \in \mathbb{C}$ and $S_{i_{k}}$ are invertible positive operators. Then

$$
T_{1} \otimes T_{2} \otimes \cdots \otimes T_{m}=\sum_{1 \leq 1_{k}, \cdots, n_{k} \leq 5}\left(m_{1_{k}} \cdots m_{n_{k}}\right) S_{1_{k}} \otimes \cdots \otimes S_{n_{k}} .
$$

Using Lemma 4.2, one can see that

$$
\gamma_{z}\left(S_{1_{k}} \otimes \cdots \otimes S_{n_{k}}\right) \in \pi(G L(n))^{\prime \prime} .
$$

Thus

$$
\gamma_{z}\left(T_{1} \otimes T_{2} \otimes \cdots \otimes T_{m}\right) \in \pi(G L(n))^{\prime \prime} .
$$

Therefore $\pi(G L n))^{\prime \prime}=\mathcal{A}$ and we complete the proof.

Remark 4.1. As a $\rho\left(S_{m}\right)-\mathcal{A}$ bimodule, $\left(\mathbb{C}^{n}\right)^{\otimes m}$ can be decomposed as

$$
\left(\mathbb{C}^{n}\right)^{\otimes m}=\sum_{\left\{\lambda: \lambda_{1} \leq n\right\}} S_{\lambda} \otimes G_{\lambda},
$$

where $\lambda=\left(\lambda_{1}, \lambda_{2}, \cdots, \lambda_{k}\right)$ is a partition of $m$, and $S_{\lambda}$ is the irreducible $S_{m}$-module corresponding to the Young diagram $\lambda$, while $G_{\lambda}$ is that of $G L(n)$ with the highest weight $\left[\lambda_{1}, \lambda_{2}, \cdots, \lambda_{k}\right]$. 


\section{ACKNOWLEDGMENT}

The author wants to thank the referee for his precious suggestions which are crucial in this paper.

\section{REFERENCES}

[1] E. Abe, Hopf Algebras, Cambridge University Press, 1977. MR 83a:16010

[2] P. Bantay, Orbifolds and Hopf Algebras, Phys. Lett. B 245, 477-479, 1990. MR 91k:81147

[3] A.Van Daele, Multiplier Hopf algebras, Trans AMS vol342, No2, 917-932, 1994. MR 94h:16075

[4] A.Van Daele, Discrete Quantum Groups, J. Algebra 180, 431-444(1996). MR 97a:16076

[5] R. Dijkgraaf, V.Pasquier, V. Roche, Talk presented at Inter. Coll. on Modern Quantum Field Theory, Tata Institute, 8-14, Jan. 1990.

[6] A. Fulton, I.Harrie, Representation Theory, GTM 129, Springer-Verlag 1991. MR 93a:20069

[7] Jiang Lining, The Schur-Weyl Duality Between Quantum Group of Type A and Hecke Algebra, Advanced in Mathematics (in Chinese), Vol29, No5, 443-456, 2000.

[8] M Jimbo, q-analogue of $U(g l(n))$, Hecke Algebra and the Yang-Baxter Equation, Lett. Math. Phys. Vol 11(3), 247-252, 1986. MR 87k:17011

[9] C. Kassel, Quantum Groups, GTM 155, Springer-Verlag, 1995. MR 96e:17041

[10] Li Bingren, Operator Algebras (in Chinese), Scientific Press, 1998.

[11] G.T.Murphy, C*-Algebras and Operator Theory, Academic Press, London, 1990. MR 91m:46084

[12] P.Podles, S.L.Woronowicz, Quantum Deformation of Lorentz Group, Comm. Math. Phys. 130, 381-441, 1990. MR 91f:46100

[13] M.E. Sweedler, Hopf Algebras, Benjamin, New York, 1969. MR 40:5705

[14] K. Szlachanyi, P. Vecsernyes, Quantum Symmetry and Braided Group Statistics in G-Spin Models, Comm. Math. Phys. 156, 127-168, 1993. MR 94m:81063

[15] H. Weyl, The Classical Groups, Princeton Mathematics Series, Princeton University Press, 1939. MR 98k:01049

Department of Mathematics, Beijing Institute of Technology, Beijing (100081), PeoPLE'S REPUBlic OF ChinA

E-mail address: jiangjln@sina.com

Department of Mathematics, Peking University, Beijing (100871), People's Republic OF CHINA

E-mail address: maguo@pku.edu.cn

Department of Mathematics, Peking University, Beijing (100871), People's Republic OF CHINA 